\title{
Glioma-Induced Disruption of Resting-State Functional Connectivity and Amplitude of Low-Frequency Fluctuations in the Salience Network
}

\author{
(D). Yang, (D) S. Gohel, (D) Z. Zhang, (D) V. Hatzoglou, (D) A.I. Holodny, and (D)B.A. Vachha
}

\begin{abstract}
BACKGROUND AND PURPOSE: Cognitive challenges are prevalent in survivors of glioma, but their neurobiology is incompletely understood. The purpose of this study was to investigate the effect of glioma presence and tumor characteristics on resting-state functional connectivity and amplitude of low-frequency fluctuations of the salience network, a key neural network associated with cognition.
\end{abstract}

MATERIALS AND METHODS: Sixty-nine patients with glioma (mean age, 48.74 [SD, 14.32] years) who underwent resting-state fMRI were compared with 31 healthy controls (mean age, 49.68 [SD, 15.54] years). We identified 4 salience network ROls: left/right dorsal anterior cingulate cortex and left/right anterior insula. Average salience network resting-state functional connectivity and amplitude of low-frequency fluctuations within the 4 salience network ROls were computed.

RESULTS: Patients with gliomas showed decreased overall salience network resting-state functional connectivity $(P=.001)$ and increased amplitude of low-frequency fluctuations in all salience network ROIs $(P<.01)$ except in the left dorsal anterior cingulate cortex. Compared with controls, patients with left-sided gliomas showed increased amplitude of low-frequency fluctuations in the right dorsal anterior cingulate cortex $(P=.002)$ and right anterior insula $(P<.001)$, and patients with right-sided gliomas showed increased amplitude of low-frequency fluctuations in the left anterior insula $(P=.002)$. Anterior tumors were associated with decreased salience network resting-state functional connectivity $(P<.001)$ and increased amplitude of low-frequency fluctuations in the right anterior insula, left anterior insula, and right dorsal anterior cingulate cortex. Patients with high-grade gliomas had decreased salience network resting-state functional connectivity compared with healthy controls $(P<.05)$. The right anterior insula showed increased amplitude of low-frequency fluctuations in patients with grade II and IV gliomas compared with controls $(P<.01)$.

CONCLUSIONS: By demonstrating decreased resting-state functional connectivity and an increased amplitude of low-frequency fluctuations related to the salience network in patients with glioma, this study adds to our understanding of the neurobiology underpinning observable cognitive deficits in these patients. In addition to more conventional functional connectivity, amplitude of low-frequency fluctuations is a promising functional-imaging biomarker of tumor-induced vascular and neural pathology.

ABBREVIATIONS: ALFF = amplitude of low-frequency fluctuations; BOLD = blood oxygen level-dependent; PCG $=$ precentral gyri; RSFC $=$ resting-state functional connectivity; rsfMRI = resting-state fMRI; $\mathrm{SN}$ = salience network

D etrimental effects of cancer on cognitive function and, consequently, on the quality of life are emerging as a key focus of cancer survivorship both in research and clinical practice. ${ }^{1,2}$

Received June 22, 2020; accepted after revision October 2.

From the Departments of Radiology (J.Y., V.H., A.I.H., B.A.V.), Epidemiology and Biostatistics (Z.Z.), and Brain Tumor Center (V.H., A.I.H., B.A.V.), Memorial Sloan Kettering Cancer Center, New York, New York; New York University Grossman School of Medicine (J.Y.), New York University, New York, New York; Department of Health Informatics (S.G.), Rutgers University School of Health Professions, Newark, New Jersey; Department of Radiology (V.H., A.I.H., B.A.V.), Weill Medical College of Cornell University, New York, New York; and Department of

Neuroscience (A.I.H.), Weill-Cornell Graduate School of the Medical Sciences, New York, New York.

J. Yang and S. Gohel contributed equally to this work.

Brain tumors have been shown to affect memory, processing, and attention in patients; however, their underlying neurobiology is incompletely understood. ${ }^{3}$ Using resting-state functional MR imaging (rsfMRI) to evaluate changes in cognitive resting-state

Supported by the National Institutes of Health/National Cancer Institute Cancer Center Support (P30 CA008748) and the National Cancer Institute of the National Institutes of Health (R25CA020449).

Please address correspondence to Behroze A. Vachha, MD, PhD, Department of Radiology, Memorial Sloan Kettering Cancer Center, 1275 York Ave, NY, NY 10065; e-mail: vachhab@mskcc.org

- Indicates open access to non-subscribers at www.ajnr.org

http://dx.doi.org/10.3174/ajnr.A6929 
networks may provide a better understanding of the pathology underlying the observable cognitive disruptions in gliomas, the most common primary brain tumor in adults.

A "triple network model" of neurocognitive pathology has been proposed, which encompasses the default mode network, involved in mind wandering; the central executive network, involved in decision-making; and the salience network ( $\mathrm{SN}$ ), implicated in modulating activation of the default mode network and central executive network by detecting the presence of salient stimuli. ${ }^{4-8}$ While previous rsfMRI research has largely focused on tumor-induced changes in the default mode network, ${ }^{9,10}$ our study examined the less-studied SN, a network rooted in the anterior insula and the dorsal anterior cingulate cortex. ${ }^{6}$

Prior studies evaluating gliomas and SN resting-state functional connectivity (RSFC) provided conflicting results in small patient samples: Maesawa et $\mathrm{al}^{10}$ found no significant differences in the $\mathrm{SN}$ in 12 patients, while Liu et $\mathrm{al}^{11}$ more recently found decreased SN connectivity in 13 patients. Gliomas impact the integrity of the neurovascular unit to varying degrees, resulting in neurovascular uncoupling that has been reported to confound fMRI interpretations in patients with brain tumors. ${ }^{12-14}$ Additionally, research has reported neuronal plasticity manifested by structural reorganization and functional remodeling of neural networks in patients with gliomas with possible alterations in clinically observable cognitive manifestations. ${ }^{15-17}$ An rsfMRI metric, the amplitude of low-frequency fluctuations (ALFF), has recently shown promise as a biomarker for brain plasticity and hemodynamic characterization, including neurovascular uncoupling in patients with gliomas. ${ }^{15-19}$

The purpose of this study was to investigate the effect of glioma presence and tumor characteristics on overall RSFC and regional normalized ALFF within the $\mathrm{SN}$ in a large patient population. We hypothesized that there would be decreased average SN RSFC and altered ALFF in patients with gliomas compared with healthy controls. Recent studies have acknowledged that gliomas have variable effects on network integrity based on lesion location and proximity to network ROIs, ${ }^{20-22}$ and unilateral gliomas can be associated with plasticity in both the ipsilateral and contralateral hemispheres. ${ }^{11,17}$ Research also supports differences in resting-state network reorganization in aggressive high-grade gliomas compared with slower-growing low-grade gliomas. ${ }^{20,23}$ Therefore, we also hypothesized that there would be differences in average SN RSFC and regional ALFF in patients based on the anterior-versus-posterior location, hemispheric side, and grade of glioma.

\section{MATERIALS AND METHODS Study Sample}

This observational, retrospective case-control study was approved by the Memorial Sloan Kettering Cancer Center institutional review board and was compliant with the Health Insurance Portability and Accountability Act. The re-quirement for written informed consent was waived due to the retrospective nature of the study. Sixty-nine patients with brain tumors referred for presurgical functional mapping by fMRI between January 2016 and April 2018 who met inclusion/exclusion criteria were included in the study. Inclusion criteria were the following: 1) older than
18 years of age, and 2) the presence of supratentorial, unilateral intra-axial gliomas confirmed by pathology after an operation. Patients were excluded from the study for the following reasons: 1) No rsfMRI was performed, or there was incomplete rsfMRI data; 2) histopathology confirmed nonglial tumors; and 3) there were documented pre-existing neuropsychological, neurodegenerative, or psychiatric comorbidities. For comparison, 31 ageand sex-matched healthy controls were selected from the Nathan Kline Institute-Rockland Sample enhanced, open-access data repository. $^{24}$

\section{Image Acquisition}

Patients underwent rsfMRI with a $3 \mathrm{~T}$ imaging unit (750 W; GE Healthcare) and a gradient-echo echo-planar imaging sequence (TR/TE, 2500/30 ms; $64 \times 64$ matrix; 4-mm section thickness; $80^{\circ}$ flip angle; 7-minute acquisitions). Images in control subjects were obtained using a 3T Magnetom Trio, A Tim System imaging unit (Siemens) and an echo-planar imaging sequence (TR/TE, 2500/30 ms; $72 \times 72$ matrix; 3 -mm section thickness; 10-minute acquisitions). T1-weighted anatomic images for patients and control subjects were obtained with spoiled gradient recalled and high-resolution 3D MPRAGE sequences. Similar to control subjects, before each rsfMRI scan, patients were instructed to keep their eyes open and stay awake. Because patients underwent both rsfMRI and task-based fMRI during the same scan sessions as part of presurgical work-up, the order of sequence acquisition was randomized to minimize confounds.

\section{rsfMRI Preprocessing}

In the current study, we implemented a data-processing scheme similar to earlier published methods. ${ }^{21}$ Analysis was performed using Statistical Parametric Mapping, Version 12 (http://www.fil. ion.ucl.ac.uk/spm/software/spm12) and Analysis of Functional Neuro Images (AFNI, Version 18.0.21; http://afni.nimh.nih.gov/ afni). ${ }^{25}$ Briefly, we removed the first 5 time points of fMRI data to allow T1-relaxation effects followed by head-motion correction and coregistration. These steps were followed by segmentation of each subject's anatomic images into gray matter, white matter, and CSF images. Deformation fields were derived to transfer functional images into Montreal Neurological Institute standard space using segment procedures in SPM12 software. Finally, we used this subject-specific deformation field to transform the functional images into standard space images. Each of the functional images normalized to standard space was then manually inspected using consensus viewing by a neuroradiologist and a neuroimaging consultant with up to 10 years of fMRI experience to ensure that there were no major distortions due to mass effect from large tumors. A general linear model-based regression approach was implemented using 24 motion regressors (6 motion parameters derived in the motion-correction step, 6 squared of the original motion parameters, 6 one-time-point delayed version of the motion parameters, and finally 6 squared of the delayedmotion parameters) to remove the effects of motion-related noise from the blood oxygen level-dependent (BOLD) fMRI data. ${ }^{26}$

Because most tumors infiltrated the boundaries of gray matter, white matter, and CSF and given the differential locations of tumors, no regressors from the CSF or white matter region were 
included in the regression model, to avoid eliminating signals of interest. Following regression, temporal filtering between the frequency bands of 0.01 and $0.1 \mathrm{~Hz}$ and spatial smoothing with a 6$\mathrm{mm}$ full width at half maximum Gaussian filter was applied. Using the head-motion parameters, we calculated subject-specific measures of mean frame-wise displacement. ${ }^{27}$ Because both patients and healthy controls demonstrated a mean frame-wise displacement of $<0.5 \mathrm{~mm}$ and no differences in mean frame-wise displacement were observed between the patient and healthy control groups $(P=.28)$, data scrubbing was not performed. Only the first 160 time points from the healthy controls were used to match the number of time points in patients with glioma.

\section{rsfMRI Data Analysis}

Four spatially distinct ROIs within the SN were identified: left dorsal anterior cingulate cortex (Montreal Neurological Institute coordinates: $-5,26,31$, right dorsal anterior cingulate cortex (Montreal Neurological Institute coordinates: 5, 26, 31), left anterior insula (Montreal Neurological Institute coordinates: $-34,17$, -4 ), and right anterior insula (Montreal Neurological Institute coordinates: $37,20,-6) .{ }^{28}$ For each region, a 6-mm sphere was created, resulting in 4 ROI masks. For each participant, the mean BOLD fMRI for each region was extracted from the preprocessed rsfMRI, and $4 \times 4$ functional connectivity matrices were derived representing functional integration within the SN. The average value across the different region-specific RSFC values was then computed to derive the average SN RSFC for each subject.

Mean normalized ALFF was calculated for each subject. ${ }^{29}$ The ALFF measure at each voxel represented the averaged square root of the power within the frequency window of $0.01-0.1 \mathrm{~Hz}$, normalized by the mean within-brain ALFF value for that subject. The mean normalized ALFF value for each of the 4 SN ROIs (right dorsal anterior cingulate cortex, left dorsal anterior cingulate cortex, right anterior insula, left anterior insula) was extracted. Identical ROIs were used for analysis of the SN RSFC and ALFF maps.

To demonstrate that there were no significant differences in scanner parameters between the patient scans and the Nathan Kline Institute control scans, we compared BOLD fMRI activation between patients and healthy controls using the bilateral precentral gyri (PCG) as a reference region. A subset of 15 patients with no tumor infiltration or mass effect in the bilateral PCG was selected, along with 15 age-matched controls from our healthy control group. A 6-mm spherical ROI mask was created for both PCG regions (left PCG, Montreal Neurological Institute coordinates: $-44,-6,49$; right PCG Montreal Neurological Institute coordinates: $44,-11,38) .{ }^{30}$ Mean BOLD fMRI signals were extracted from each of the subject's preprocessed rsfMRI using these ROI masks, and RSFC between the right and left PCG BOLD fMRI signal was calculated. Mean normalized ALFF values were also extracted for the left and right PCG.

\section{Statistical Analysis}

Statistical analyses were performed with Matlab 9.4 and the Statistics and Machine Learning Toolbox 11.3 (release 2018a; MathWorks) with statistical significance defined by $P<.05$.
Independent 2-sample $t$ tests were used to evaluate the overall effect of glioma presence on the average SN RSFC and ROI-specific ALFF between patients and healthy controls. ANCOVA with age, sex, and handedness as covariates was used to analyze the effect of glioma location (anterior versus posterior), glioma side (right versus left), and glioma grade on average SN RSFC and ROI-specific ALFF between patients and healthy controls followed by a post hoc Tukey-Kramer test for multiple simultaneous pair-wise comparisons. To correct for multiple comparisons, we calculated the Bonferroni-adjusted significance level of .01 to account for the increased possibility of type I error for the 2-sample $t$ tests and ANCOVA. Two 1-sided tests were used to calculate the equivalence among scanner parameters in a subset of patient and Nathan Kline Institute control scans with the upper and lower equivalence bounds set at a positive or negative difference of $d=0.5^{31}$

\section{RESULTS}

\section{Sample Characteristics}

Sixty-nine patients (mean age, 48.74 [SD, 14.32] years; range, 1977 years; 45 men) and 31 healthy controls (mean age, 49.68 [SD, 15.54 ] years; range, $22-68$ years; $18 \mathrm{men})$ were included in this study. No differences were observed in age $(P=.7685)$, sex $\left(\chi^{2}=\right.$ $0.47, P=.49)$, and handedness $\left(\chi^{2}=0.63, P=.43\right)$ between the patient and healthy control groups. The 69 patients were grouped into the following; 1$)$ patients with anterior $(n=53)$ and posterior $(n=16)$ gliomas; 2$)$ patients with left-sided $(n=41)$ and right-sided $(n=28)$ gliomas; and 3$)$ patients with grade II $(n=25)$, III $(n=17)$, and IV $(n=27)$ gliomas. For the purposes of this study, "anterior" gliomas were located in the frontal or anterior temporal lobes and "posterior" gliomas were located in the parietal, occipital, or posterior temporal lobes, as classified by radiology report review and manually confirmed using consensus review by 2 neuroradiologists with up to 20 years of experience. Glioma grade was based on the histopathology of tumor biopsies categorized by the 2016 World Health Organization Classification of Central Nervous System Tumors. ${ }^{32}$ Demographic information and tumor characteristics are summarized in Table 1.

Of the 69 patients, 41 (59\%) patients had newly diagnosed gliomas (18 with grade II, 8 with grade III, and 15 with grade IV gliomas) and 28 (41\%) had recurrent gliomas (7 with grade II, 9 with grade III, and 12 with grade IV gliomas). Although the average SN RSFC was slightly lower in patients with recurrent gliomas (mean $\pm[S D, 0.55 \pm 0.23]$ ) compared with newly diagnosed gliomas (mean $\pm \mathrm{SD}, 0.56 \pm 0.27]$ ), there were no significant differences between the 2 groups $(P=.89)$. Similarly, there were no significant differences in regional ALFF between the newly diagnosed gliomas and the recurrent gliomas (all, $P>.05$ ). When they were stratified by grade, there were no significant differences in average SN RSFC or ALFF between recurrent gliomas-versusde novo gliomas (all, $P>.05$ ).

For the subset of patients with no involvement of the bilateral PCGs by tumor and their age-matched controls, RSFC between the right and left PCG and regional ALFF for each of the right and left PCGs between patient and Nathan Kline Institute control 
scans was equivalent, on the basis of equivalence bounds of -0.5 and 0.5

\section{Overall Effect of Glioma Presence on the RSFC and ROI- Specific ALFF of the SN}

Patients with gliomas demonstrated lower average SN RSFC compared with healthy controls $(P=.001)$. We observed increased ALFF in patients with gliomas compared with healthy controls in the right dorsal anterior cingulate cortex $(P<.001)$, left anterior insula $(P=.001)$, and right anterior insula $(P<.001)$. Results are reported in Table 2. Maps of RSFC and ALFF for a representative patient and healthy control are illustrated in Fig 1.

\section{Effect of Glioma Anteriority on RSFC and ROI-Specific ALFF of the SN}

We observed a significant effect of anterior-versus-posterior tumor location on average SN RSFC $(F[2,94]=7.09, P=.001)$. Post hoc Tukey comparison showed lower SN RSFC in patients

Table 1: Study group and tumor characteristics

\begin{tabular}{|c|c|c|}
\hline Sample Characteristics & $\begin{array}{l}\text { Patients } \\
(n=69)\end{array}$ & $\begin{array}{l}\text { Healthy Controls } \\
\qquad(n=31)\end{array}$ \\
\hline \multicolumn{3}{|l|}{ Sex } \\
\hline Male & 45 & 18 \\
\hline Female & 24 & 13 \\
\hline \multicolumn{3}{|l|}{ Age (yr) } \\
\hline Mean & $48.74[S D, 14.32]$ & $49.68[S D, 15.54]$ \\
\hline Range & $19-77$ & $22-68$ \\
\hline \multicolumn{3}{|l|}{ Handedness ${ }^{a}$} \\
\hline Right & 61 & 29 \\
\hline Left & 8 & 2 \\
\hline \multicolumn{3}{|l|}{ Glioma location ${ }^{\mathrm{b}}$} \\
\hline Anterior & 53 & \\
\hline Posterior & 16 & \\
\hline \multicolumn{3}{|l|}{ Glioma hemisphere } \\
\hline Left & 41 & \\
\hline Right & 28 & \\
\hline \multicolumn{3}{|l|}{$\begin{array}{l}\text { Glioma grade } \\
\text { (WHO classification) }\end{array}$} \\
\hline Grade II & 25 & \\
\hline Grade III & 17 & \\
\hline Grade IV & 27 & \\
\hline Antiepileptics (yes/no) & $55 / 14$ & \\
\hline Steroids (yes/no) & $33 / 36$ & \\
\hline
\end{tabular}

Note:-WHO indicates World Health Organization.

${ }^{a}$ Handedness was determined by the Edinburgh Handedness Inventory.

b"Anterior" tumors are located in the frontal or anterior temporal lobes.

"Posterior" tumors are located in the parietal or posterior temporal lobes, as classified by radiology report review.

Table 2: Effect of glioma presence on SN RSFC and regional ALFF

\begin{tabular}{lllc}
\hline \multicolumn{1}{c}{ Analysis } & Healthy Controls & Patients with Gliomas & $\boldsymbol{P}$ Values \\
\hline Average SN RSFC & $0.73[\mathrm{SD}, 0.20]$ & $0.56[\mathrm{SD}, 0.25]$ & $.001^{\mathrm{b}}$ \\
ALFF & & & \\
L-dACC & $0.93[\mathrm{SD}, 0.16]$ & $0.98[\mathrm{SD}, 0.22]$ & .21 \\
R-dACC & $0.93[\mathrm{SD}, 0.16]$ & $1.08[\mathrm{SD}, 0.22]$ & $<.001^{\mathrm{b}}$ \\
L-AINS & $0.76[\mathrm{SD}, 0.12]$ & $0.90[\mathrm{SD}, 0.23]$ & $.001^{\mathrm{b}}$ \\
R-AINS & $0.87[\mathrm{SD}, 0.13]$ & $1.13[\mathrm{SD}, 0.32]$ & $<.001^{\mathrm{b}}$ \\
\hline
\end{tabular}

Note:-L-dACC indicates left dorsal anterior cingulate cortex; R-dACC, right dorsal anterior cingulate cortex; R-AINS, right anterior insula; L-AINS, left anterior insula.

${ }^{a}$ Data are presented as means.

${ }^{\mathrm{b}} \mathrm{P}<.01$ after Bonferroni-correction. with anteriorly located gliomas compared with healthy controls $(P<.001)$. No significant difference was found in patients with posteriorly located gliomas $(P=.44)$ compared with healthy controls. No significant differences were observed when comparing the patient subgroup with anteriorly located gliomas with those with posteriorly located gliomas $(P=.26)$.

There was a significant effect of tumor location on ALFF values for right dorsal anterior cingulate cortex $(F[2,94]=5.89$, $P=.004)$, left anterior insula $(F[2,94]=5.18, P=.0074)$, and right anterior insula $(F[2,94]=10.12, P<.001)$. Post hoc comparisons showed that compared with healthy controls, patients with anterior tumors demonstrated increased ALFF in the right dorsal anterior cingulate cortex $(P=.009)$, left anterior insula $(P=.008)$, and right anterior insula $(P=.002)$, while patients with posterior tumors demonstrated increased ALFF in the right dorsal anterior cingulate cortex $(P=.016)$ and right anterior insula $(P<.001)$. There was no significant difference in ALFF between patients in the anterior and posterior tumor groups (Fig 2).

\section{Effect of Glioma Hemispheric Side on RSFC and ROI-Specific ALFF of the SN}

An ANCOVA comparing patients with left-sided gliomas, rightsided gliomas, and controls showed significant differences in average SN RSFC $(F[2,94]=5.70, P=.005)$. Post hoc comparisons showed patients in both left-sided $(P=.007)$ and right-sided $(P=.02)$ glioma groups had lower SN RSFC compared with controls. Comparison among the patient subgroups, however, showed no significant effects of glioma sidedness on average SN $\operatorname{RSFC}(P=.99)$.

ANCOVA analysis showed a significant association between glioma sidedness and ALFF in the right dorsal anterior cingulate cortex $(F[2,94]=6.37, P=.003)$, left anterior insula $(F[2,94]=6.28, P=.003)$, and right anterior insula $(F[2,94]=15.09, P<.001)$. Post hoc comparisons showed that compared with healthy controls, patients with tumors in the left hemisphere demonstrated increased ALFF in the right dorsal anterior cingulate cortex $(P=.002)$ and right anterior insula $(P<.001)$, while patients with right-sided tumors demonstrated increased ALFF in the left anterior insula $(P=.002)$. Within-group comparison showed increased ALFF in right anterior insula in patients with left-sided tumors compared with those with right-sided tumors $(P=.004)$. Results are illustrated in Fig 2.

\section{Effect of Tumor Grade on RSFC and ROI-Specific ALFF of the SN}

Tumor grade was associated with decreased average SN RSFC $(F[3,93]=5.03, P=.003)$. Post hoc comparisons showed decreased average SN RSFC in patients with grade III $(P=.004)$ and grade IV tumors $(P=.017)$ compared with healthy controls.

Tumor grade was associated with increased ALFF within the left anterior insula $(F[3,93]=4.48, P=.006)$ and right anterior insula $(F[3,93]=5.97, P<.001)$. Post hoc comparisons showed increased ALFF in the right anterior insula in patients with grade II 


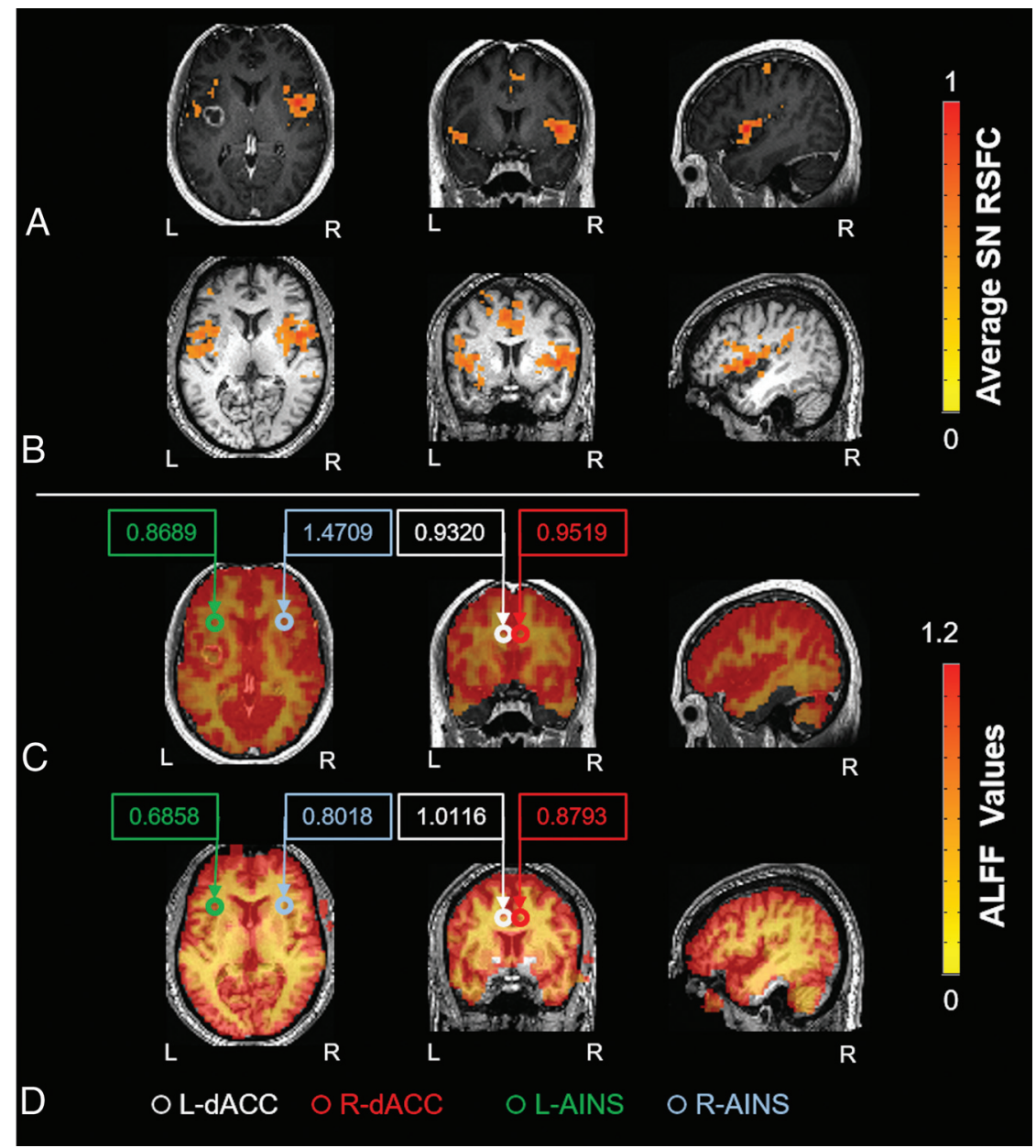

FIG 1. Map of functional connectivity and amplitude of low-frequency fluctuations in the SN Decreased functional connectivity within the SN was seen by placing a seed in the R-dACC in a representative patient with a left frontal glioblastoma $(A)$ and a healthy control $(B)$. ALFF map of the same patient $(C)$ and a healthy control $(D)$. Circles represent the 4 SN ROIs. Values in boxes represent average ALFF values within the specific ROIs. Increased ALFF values were seen in all 4 SN ROIs in the patient compared with the healthy control. R-dACC indicates right dorsal anterior cingulate cortex; L-dACC, left dorsal anterior cingulate cortex; L-AINS, left anterior insula; RAINS, right anterior insula; L, left; R, right.

$(P=.006)$ and IV $(P=.003)$ tumors and trend-level differences in patients with grade III tumors $(P=.08)$ compared with healthy controls (Fig 3).

\section{DISCUSSION}

Our study investigated resting-state fMRI alterations in the $\mathrm{SN}$ in a large sample of patients with gliomas and produced 3 main findings: First, the average SN RSFC was decreased in patients with gliomas compared with healthy controls. Second, disruptions in ALFF were found in established SN ROIs. Third, tumor location and grade produced differential effects on SN functional connectivity and ALFF.

The SN functions to select meaningful stimuli from an array of sensory input. ${ }^{33}$ At a regional level, patients with gliomas showed disrupted ALFF in 3 of the 4 SN-specific ROIs (right anterior insula, left anterior insula, and right dorsal anterior cingulate cortex) compared with healthy controls. The most significant change was seen in the right anterior insula, which has specifically been proposed as a "causal outflow hub" driving the switch between the default mode network and central executive network, with anterior insula dysfunction and consequently impaired network switching implicated in other disorders demonstrating impaired executive control. ${ }^{4,34,35}$ Our findings of globally decreased SN RSFC and compromised integrity of key $\mathrm{SN}$ regions in patients with glioma may explain several of the clinically documented cognitive challenges that these patients experience. ${ }^{3}$

Consistent with previous research, ALFF values in the tumor-contralateral SN ROIs were increased compared with ipsilateral regions in our study. ${ }^{17,18,36}$ The relatively increased ALFF in the lesion-contralateral ROIs could be explained not only by truly asymmetric neural activity but also by neurovascular uncoupling in the tumor ipsilateral hemisphere. This tumorinduced loss of neurovascular autoregulation can potentially alter the vascular component of BOLD signal to a degree that confounds measurement of neural activity. ${ }^{14,18,37,38}$ The observation of increased ALFF in SN ROIs of patients with tumor compared with controls and in tumor-contralateral compared with tumor-ipsilateral regions could also be explained by functional compensation for injured tissue due to neural plasticity, which may manifest as altered metabolism or blood flow. ${ }^{17,39}$ Future studies should investigate changes in ALFF measured across the whole brain, to study whether there are paradoxic effects on BOLD activation in areas proximal or distal to the tumor produced by a combination of neurovascular uncoupling and functional compensation.

Not unexpectedly, tumors in the frontal or anterior temporal lobes of patients, closer to key $\mathrm{SN}$ regions, were associated with lower SN RSFC compared with healthy controls, while posterior tumors showed no significant differences in connectivity. Higher glioma grades were associated with decreased overall SN RSFC compared with controls, possibly due to a combination of factors including true-positive changes due to increased tumor infiltration and disrupted functional reorganization, ${ }^{9,20}$ as well as false-negative signals due to tumorinduced neurovascular uncoupling that may have artifactually decreased RSFC due to altered hemodynamics. ${ }^{22,36}$ These 


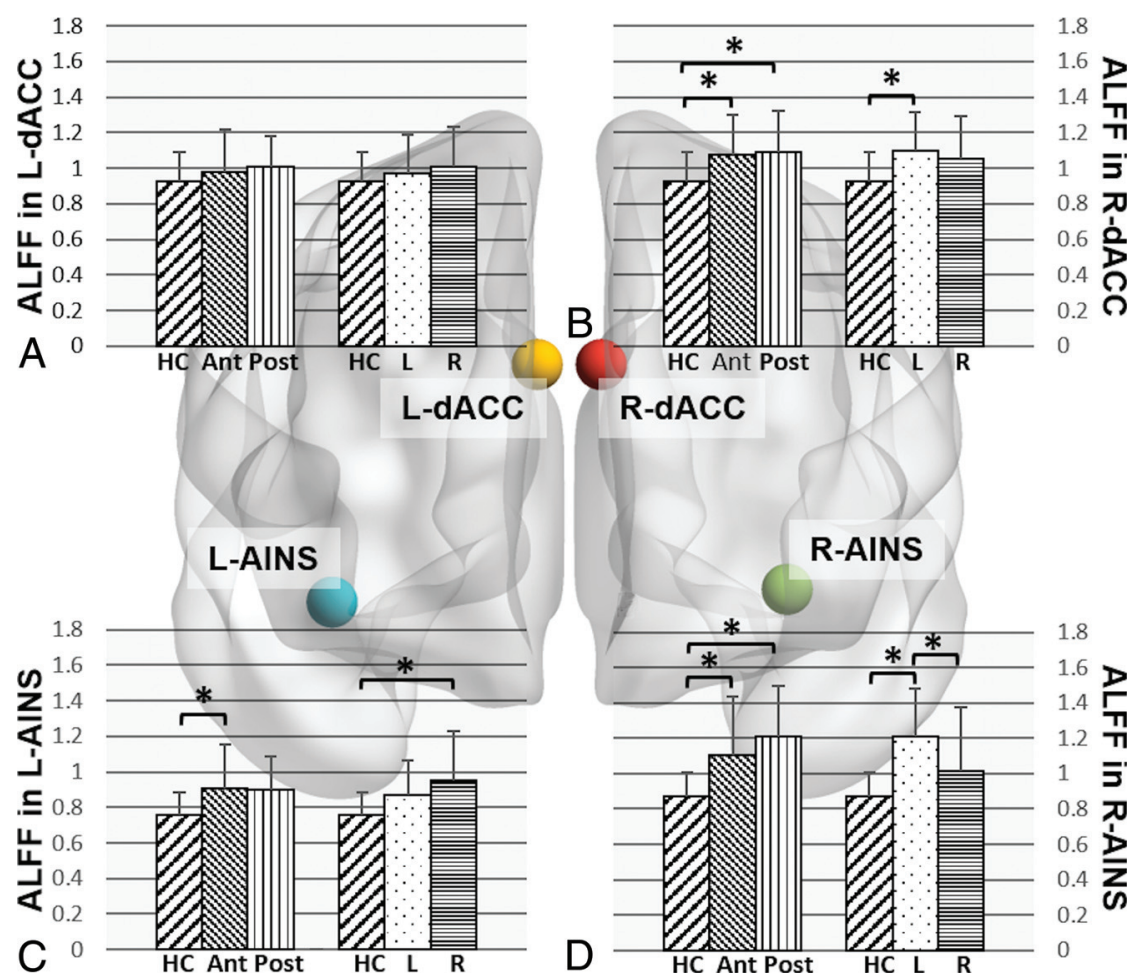

FIG 2. Effect of glioma location on amplitude of low-frequency fluctuations in the SN ROIs. Bar graphs for comparison of mean ALFF values in the L-dACC (A), R-dACC (B), L-AINS (C), and RAINS $(D)$ in healthy controls and patients grouped by glioma location. $\mathrm{HC}$ indicates healthy controls; L, patients with gliomas in left hemisphere; R, patients with gliomas in right hemisphere; Ant, patients with anteriorly located gliomas; Post, patients with posteriorly located gliomas; L$\mathrm{dACC}$, left dorsal anterior cingulate cortex; R-dACC, right dorsal anterior cingulate cortex; LAINS, left anterior insula; R-AINS, right anterior insula. The asterisk indicates $P<.05$ post hoc Tukey-Kramer test for multiple comparisons.

differences than fractional ALFF. $^{42}$ However, both indexes reflect different aspects of low-frequency oscillation amplitude with complementary strengths and weaknesses in the detection of intrinsic brain activity, ${ }^{42}$ and future work that includes both measures of low-frequency oscillations may be useful to characterize the glioma-associated neurophysiologic alterations.

Our study showed no statistically significant differences in average SN RSFC and ALFF between patients with newly diagnosed gliomas and those with recurrent gliomas, even after stratifying for differences in tumor grade, though patients with recurrent gliomas demonstrated slightly decreased average SN RSFC compared with those with newly diagnosed gliomas. Although this countered our expectation of possible decreased functional connectivity in patients with recurrent glioma due to prior operative and chemoradiation treatment, the smaller sample size of the recurrent glioma group may have reduced the power to detect functional differences beyond the findings reported here. In addition, other important factors such as variability in chemotherapeutic regimens, differences in duration and total dosage of radiation therapy, use of concomitant steroids and antiepi-

findings warrant cautious clinical interpretation of rsfMRI findings in high-grade gliomas.

In the present study, we observed opposite effects of tumor on measures of resting-state brain function: The RSFC of the SN was significantly reduced, while ALFF measures were significantly increased in patients with tumor compared with healthy controls. While previous research in healthy controls reported a positive correlation between ALFF values and overall functional connectivity of specific brain regions, ${ }^{40}$ we suggest the presence of a complex relationship between regional ALFF values and overall RSFC in patients with tumors, likely due to a combination of tumor-induced differences in vascularization, blood flow, and altered neurovascular coupling. ${ }^{15}$ These results need further investigation and correlation with perfusion parameters to determine the role of ALFF as a biomarker of tumor vascularity in patients with gliomas.

In addition to ALFF, fractional ALFF is a frequency-normalized index of ALFF that corrects for broad-range frequency variations and suppresses vascular contributions. ${ }^{41}$ In the present study, we focused on mean normalized ALFF instead of fractional ALFF because of its reported increased reliability within gray matter regions compared with fractional ALFF and, thus, its potential to be more sensitive to discern group leptic drugs, as well as differences in genomic mutation would need to be accounted for as well. While beyond the scope of the current study, they merit further exploration in a larger study.

A few limitations to our study warrant further investigation: First, our healthy control group was obtained from an openaccess data base that had similar-but-not identical acquisition parameters compared with our patient group. However, previous research has demonstrated that resting-state networks are stable across datasets collected using different scanners and acquisition parameters. ${ }^{43}$ Additionally, we demonstrated equivalence in RSFC and ALFF between matched controls and a subset of our patients with no bilateral PCG tumor involvement, suggesting that the differences we observed were largely driven by underlying changes related to tumor presence, while acknowledging that variance related to differences in acquisition parameters may potentially confound interpretation of ALFF regional differences in the tumor group. Future studies using the same scanner to acquire data for patient and control groups will help address potential variances due to differences in acquisition parameters. Second, although our study demonstrated regional alterations in ALFF, these alterations may not be specific to neurovascular uncoupling or functional remodeling and may be indicative 


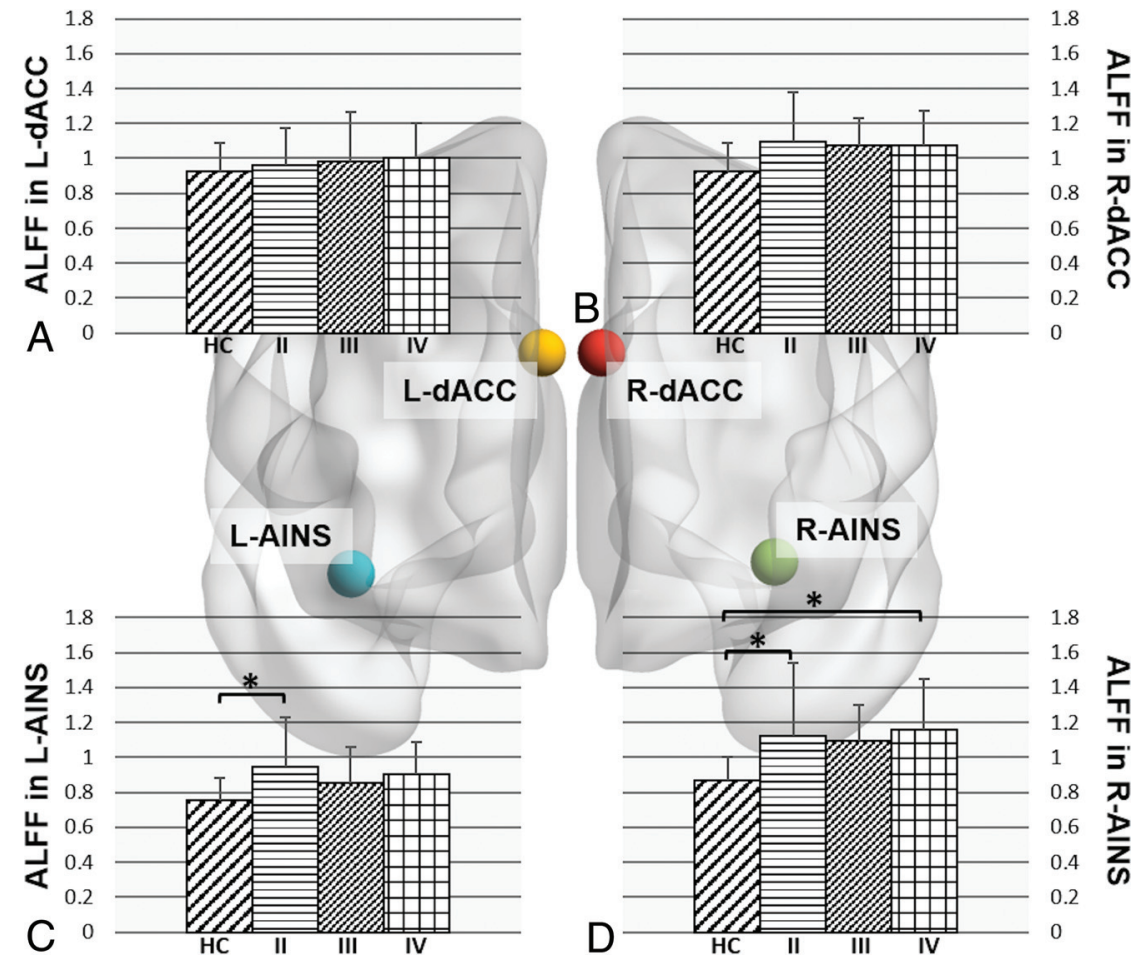

FIG 3. Effect of glioma grade on the amplitude of low-frequency fluctuations in the SN ROIs. Bar graphs for comparison of mean ALFF values in the L-dACC (A), R-dACC) (B), L-AINS (C), and RAINS $(D)$ in healthy controls and patients grouped by glioma grade. $\mathrm{HC}$ indicates healthy controls; II, patients with grade II gliomas; III, patients with grade III gliomas; IV, patients with grade IV gliomas; L-dACC, left dorsal anterior cingulate cortex; R-dACC, right dorsal anterior cingulate cortex; L-AINS, left anterior insula; R-AINS, right anterior insula.. The asterisk indicates $P<.05$ post hoc Tukey-Kramer test for multiple comparisons. patients with gliomas. In addition to more conventional functional connectivity, ALFF is a promising functional imaging biomarker of vascular and neural pathology and provides additional data to enable understanding of tumor-related disturbances of neural networks.

\section{ACKNOWLEDGMENTS}

The authors would like to thank Dr Krishna Juluru, Director of Radiology Informatics, and team, including Pierre Elnajjar and Ramon Sosa, for assistance with data management. We thank our MR imaging technologists, especially Thomas Mair and Regina Loccisano, as well as our scheduler, Anna Katz, for assistance with scanning and scheduling patients.

Disclosures: Jackie Yang-none. Andrei I. Holodny-UNRELATED: Other: fMRI Consulting, Comments: a purely educational entity. Behroze A. Vachha-none.

\section{REFERENCES}

1. Schagen SB, Klein M, Reijneveld JC, et al. Monitoring and optimising cognitive function in cancer patients: present knowledge and future directions. EJC Suppl 2014;12:29-24 CrossRef Medline

of an inherent property of the tumor itself. Adding a measure of cerebrovascular reactivity with breath-holding or gas-inhalation paradigms could further assess the degree of gliomaassociated neurovascular uncoupling, ${ }^{12,44}$ and future research is, therefore, warranted on how ALFF and neurovascular uncoupling should be accounted for in measuring rsfMRI results. Third, the clinical significance of these group-level differences at the single-subject level needs validation. Fourth, most of our patients, irrespective of whether they were newly diagnosed or had recurrent gliomas, had received antiepileptic drugs and/or steroids by the time of their rsfMRI scan, which may have contributed to the functional alterations; because only 7 patients were treatment-naïve and due to the variability in dosage and treatment durations among those who received treatment, we did not conduct further analyses on the differential effects of these treatments. Finally, future studies would also benefit from a neurocognitive measure, which could show how changes in SN functional connectivity correlate with cognitive performance.

\section{CONCLUSIONS}

By using both functional connectivity and a frequency-domain metric related to the $\mathrm{SN}$, this study adds to the understanding of neurobiologic effects on cognitive function in
2. Taphoorn MJ, Klein M. Cognitive deficits in adult patients with brain tumours. Lancet Neurol 2004;3:159-68 CrossRef Medline

3. Bergo E, Lombardi G, Guglieri I, et al. Neurocognitive functions and health-related quality of life in glioblastoma patients: a concise review of the literature. Eur J Cancer Care (Engl) 2019;28:e12410 CrossRef Medline

4. Uddin LQ. Salience processing and insular cortical function and dysfunction. Nat Rev Neurosci 2015;16:55-61 CrossRef Medline

5. Goulden N, Khusnulina A, Davis NJ, et al. The salience network is responsible for switching between the default mode network and the central executive network: replication from DCM. Neuroimage 2014;99:180-90 CrossRef Medline

6. Seeley WW, Menon V, Schatzberg AF, et al. Dissociable intrinsic connectivity networks for salience processing and executive control. J Neurosci 2007;27:2349-56 CrossRef Medline

7. Menon V. Large-scale brain networks and psychopathology: a unifying triple network model. Trends Cogn Sci 2011;15:483-506 CrossRef Medline

8. Chand GB, Wu J, Hajjar I, et al. Interactions of the salience network and its subsystems with the default-mode and the central-executive networks in normal aging and mild cognitive impairment. Brain Connect 2017;7:401-12 CrossRef Medline

9. Esposito R, Mattei PA, Briganti C, et al. Modifications of defaultmode network connectivity in patients with cerebral glioma. PLoS One 2012;7:e40231 CrossRef Medline

10. Maesawa S, Bagarinao E, Fujii M, et al. Evaluation of resting state networks in patients with gliomas: connectivity changes in the unaffected side and its relation to cognitive function. PLoS One 2015;10:e0118072 CrossRef Medline 
11. Liu D, Hu X, Liu Y, et al. Potential intra- or cross-network functional reorganization of the triple unifying networks in patients with frontal glioma. World Neurosurg 2019;128:e732-43 CrossRef Medline

12. Pillai JJ, Zaca D. Clinical utility of cerebrovascular reactivity mapping in patients with low grade gliomas. World J Clin Oncol 2011;2:397-403 CrossRef Medline

13. Pak RW, Hadjiabadi DH, Senarathna J, et al. Implications of neurovascular uncoupling in functional magnetic resonance imaging (fMRI) of brain tumors. J Cereb Blood Flow Metab 2017;37:3475-87 CrossRef Medline

14. Hou BL, Bradbury M, Peck KK, et al. Effect of brain tumor neovasculature defined by $\mathrm{rCBV}$ on BOLD fMRI activation volume in the primary motor cortex. Neuroimage 2006;32:489-97 CrossRef Medline

15. Gupta L, Gupta RK, Postma AA, et al. Advanced and amplified BOLD fluctuations in high-grade gliomas. J Magn Reson Imaging 2018;47:1616-25 CrossRef Medline

16. Zhang N, Xia M, Qiu T, et al. Reorganization of cerebro-cerebellar circuit in patients with left hemispheric gliomas involving language network: a combined structural and resting-state functional MRI study. Hum Brain Mapp 2018;39:480219 CrossRef Medline

17. Liu D, Chen J, Hu X, et al. Contralesional homotopic functional plasticity in patients with temporal glioma. J Neurosurg 2020 Jan 10. [Epub ahead of print]CrossRef Medline

18. Agarwal S, Lu H, Pillai JJ. Value of frequency domain resting-state functional magnetic resonance imaging metrics amplitude of lowfrequency fluctuation and fractional amplitude of low-frequency fluctuation in the assessment of brain tumor-induced neurovascular uncoupling. Brain Connect 2017;7:382-89 CrossRef Medline

19. Agarwal S, Sair HI, Airan R, et al. Demonstration of brain tumorinduced neurovascular uncoupling in resting-state fMRI at ultrahigh field. Brain Connect 2016;6:267-72 CrossRef Medline

20. Harris RJ, Bookheimer SY, Cloughesy TF, et al. Altered functional connectivity of the default mode network in diffuse gliomas measured with pseudo-resting state fMRI. J Neurooncol 2014;116:373-79 CrossRef Medline

21. Gohel S, Laino ME, Rajeev-Kumar G, et al. Resting-state functional connectivity of the middle frontal gyrus can predict language lateralization in patients with brain tumors. AJNR Am J Neuroradiol 2019;40:319-25 CrossRef Medline

22. Sun $\mathrm{H}$, Vachha B, Laino ME, et al. Decreased hand motor restingstate functional connectivity in patients with glioma: analysis of factors including neurovascular uncoupling. Radiology 2020;294:610-21 CrossRef Medline

23. Zhang $\mathrm{H}$, Shi $\mathrm{Y}$, Yao $\mathrm{C}$, et al. Alteration of the intra- and crosshemisphere posterior default mode network in frontal lobe patients with glioma. Sci Rep 2016;6:26972 CrossRef Medline

24. Nooner KB, Colcombe SJ, Tobe RH, et al. The NKI-Rockland Sample: a model for accelerating the pace of discovery science in psychiatry. Front Neurosci 2012;6:152 CrossRef Medline

25. Cox RW. AFNI: software for analysis and visualization of functional magnetic resonance neuroimages. Comput Biomed Res 1996;29:162-73 CrossRef Medline

26. Friston KJ, Williams S, Howard R, et al. Movement-related effects in fMRI time-series. Magn Reson Med 1996;35:346-55 CrossRef Medline

27. Jenkinson M, Bannister P, Brady $M$, et al. Improved optimization for the robust and accurate linear registration and motion correction of brain images. Neuroimage 2002;17:825-41 CrossRef Medline
28. Baur V, Hanggi J, Langer $\mathrm{N}$, et al. Resting-state functional and structural connectivity within an insula-amygdala route specifically index state and trait anxiety. Biol Psychiatry 2013;73:85-92 CrossRef Medline

29. Gohel S, Gallego JA, Robinson DG, et al. Frequency specific resting state functional abnormalities in psychosis. Hum Brain Mapp 2018;39:4509-18 CrossRef Medline

30. Dosenbach NU, Nardos B, Cohen AL, et al. Prediction of individual brain maturity using fMRI. Science 2010;329:1358-61 CrossRef Medline

31. Schuirmann DJ. A comparison of the two one-sided tests procedure and the power approach for assessing the equivalence of average bioavailability. J Pharmacokinet Biopharm 1987;15:657-80 CrossRef Medline

32. Louis DN, Perry A, Reifenberger G, et al. The 2016 World Health Organization Classification of Tumors of the Central Nervous System: a summary. Acta Neuropathol 2016;131:803-20 CrossRef Medline

33. Menon V, Uddin LQ. Saliency, switching, attention and control: a network model of insula function. Brain Struct Funct 2010;214:65567 CrossRef Medline

34. Uddin LQ, Nomi JS, Hebert-Seropian B, et al. Structure and function of the human insula. J Clin Neurophysiol 2017;34:300-06 CrossRef Medline

35. Sridharan D, Levitin DJ, Menon V. A critical role for the right fronto-insular cortex in switching between central-executive and default-mode networks. Proc Natl Acad Sci U S A 2008;105:1256974 CrossRef Medline

36. Agarwal S, Sair HI, Yahyavi-Firouz-Abadi N, et al. Neurovascular uncoupling in resting state fMRI demonstrated in patients with primary brain gliomas. J Magn Reson Imaging 2016;43:620-26 CrossRef Medline

37. Hadjiabadi DH, Pung L, Zhang J, et al. Brain tumors disrupt the resting-state connectome. Neuroimage Clin 2018;18:279-89 CrossRef Medline

38. Holodny AI, Schulder M, Liu WC, et al. Decreased BOLD functional MR activation of the motor and sensory cortices adjacent to a glioblastoma multiforme: implications for image-guided neurosurgery. AJNR Am J Neuroradiol 1999;20:609-12 Medline

39. Cheng X, Gao PY. Abnormal neural activity in children with diffuse intrinsic pontine glioma had manifested deficit in behavioral inhibition: a resting-state functional MRI study. J Comput Assist Tomogr 2019;43:547-52 CrossRef Medline

40. Di X, Kim EH, Huang CC, et al. The influence of the amplitude of low-frequency fluctuations on resting-state functional connectivity. Front Hum Neurosci 2013;7:118 CrossRef Medline

41. Zou QH, Zhu CZ, Yang Y, et al. An improved approach to detection of amplitude of low-frequency fluctuation (ALFF) for resting-state fMRI: fractional ALFF. J Neurosci Methods 2008;172:137141 CrossRef Medline

42. Zuo XN, Di Martino A, Kelly C, et al. The oscillating brain: complex and reliable. Neuroimage 2010;49:1432-45 CrossRef Medline

43. Biswal BB, Mennes M, Zuo XN, et al. Toward discovery science of human brain function. Proc Natl Acad Sci U S A 2010;107:4734-39 CrossRef Medline

44. Liu P, Welch BG, Li Y, et al. Multiparametric imaging of brain hemodynamics and function using gas-inhalation MRI. Neuroimage 2017;146:715-23 CrossRef Medline 